\title{
A Clinical Predictive Model of Progesterone Elevation on the Day of HCG Administration Was Establish Based on the Basal P, BMI and Ovarian Induction Regimen
}

chunmei yu

Changzhou Women and Children's Hospital

chao zhou

Changzhou Women and Children's Hospital

xiuliang dai

Changzhou Women and Children's Hospital

jianmei zhou

Changzhou Women and Children's Hospital

haiyan yang

Changzhou Women and Children's Hospital

yufeng wang

Changzhou Women and Children's Hospital

li Chen ( $\square$ czfychenli@126.com )

Changzhou Women and Children's Hospital

\section{Research}

Keywords: Progesterone elevation (PE), Basal P, BMI, Nomogram, Predictive model

Posted Date: September 15th, 2021

DOl: https://doi.org/10.21203/rs.3.rs-880096/v1

License: (c) (1) This work is licensed under a Creative Commons Attribution 4.0 International License.

Read Full License 


\section{Abstract}

Background The progesterone elevation (PE) on the day of human chorionic gonadotrophin ( $\mathrm{hCG}$ ) is associated with a significant decrease in the probability of clinical pregnancy after fresh embryo transfer. The goal of this study was to develop a nomogram to explore the baseline indexes to predict the occurrence of PE $(0.9 \mathrm{ng} / \mathrm{ml})$ on the day of HCG administration before IVF/ICSI treatment.

Methods The patients who were performed a GnRH agonist or antagonist pituitary suppression protocol during controlled ovulation stimulation(COS) in reproductive center of Changzhou maternal and child health care hospital from 2017-2019 were included. The nomogram was built from all participants.

Results Three variates significantly associated with the PE occurrence on the HCG administration of infertility women were the type ovulation regiment, basal $\mathrm{P}$ and BMI. This predictive model showed good calibration and discriminatory abilities, with an area under the curve (AUC) of $0.639 \varangle 95 \% \mathrm{Cl} 0.596 \sim 0.681 \rrbracket$. Hosmer and Leme show test were performed for evaluating both calibration and discrimination and confirmed our nomogram is a user-friendly graphical representation of the model $\left(\chi^{2}=4.750 \otimes P=0.784\right)$

Conclusion The nomogram presents graphically association factors (type ovulation regiment, basal $\mathrm{P}$ and $\mathrm{BMI}$ ) and prediction models which can offer simple and useful guidance to avoid the PE occurrence on the HCG administration for clinicians and infertility patients.

\section{Plain English Summary}

The progesterone elevation (PE) on the day of human chorionic gonadotrophin (hCG) has attracted a lot of attention in recent years and PE is associated with a significant decrease in the probability of clinical pregnancy after fresh embryo transfer. At present the predictors of PE occurrence are still controversial, the goal of this study was to explore the baseline indexes to predict the occurrence of $P E(0.9 \mathrm{ng} / \mathrm{ml})$ on the day of HCG before IVF/ICSI treatment.

The study project and the protocols were approved by the Ethics Committee of the Changzhou maternal and child health care hospital. The sighed informed consent was obtained from all patients.

Patients (2698 cycles) were recruited from the IVF Unit of Changzhou maternal and child health care hospital in 2017-2019. The patients were performed a GnRH antagonist or agonist pituitary suppression protocol (GnRHa long Protocol and Ultra-long Protocol).

Three variates significantly associated with the PE occurrence on the HCG administration of infertility women were the type ovulation regiment, basal $\mathrm{P}$ and $\mathrm{BMI}$. This predictive model showed good calibration and discriminatory abilities, with an area under the curve (AUC) of $0.639 \otimes 95 \% \mathrm{Cl} 0.596 \sim 0.681 \rrbracket$. Hosmer and Leme show test were performed for evaluating both calibration and discrimination and confirmed our nomogram is a user-friendly graphical representation of the model $\left(\chi^{2}=4.750 \otimes P=0.784\right)$. 
In conclusion; the nomogram presents graphically association factors (type ovulation regiment, basal $\mathrm{P}$ and $\mathrm{BMI}$ ) and prediction models which can offer simple and useful guidance to avoid the PE occurrence on the HCG administration for clinicians and infertility patients.

\section{Background}

The progesterone elevation (PE) on the day of human chorionic gonadotrophin (hCG) has attracted a lot of attention in recent years and $\mathrm{PE}$ is associated with a significant decrease in the probability of clinical pregnancy after fresh embryo transfer, estimated to be about $10 \%$ [1]. It is controversial whether PE affects clinical outcome mainly by endometrial or embryo. At present most of researchers has been in favor to support PE impacting on the endometrial receptivity rather than decreasing embryo quality $[2,3]$. More recently microarray-based studies confirmed that the PE induced a change in endometrial gene expression $[4,5]$.

The clinical significance of the effect is not negligible and for this reason several strategies have been brought up to prevent PE occurrence, and one of the most popular strategies is to freeze all embryos that were transferred for a frozen-thawed cycle subsequently[6, 7]. However, if possible, clinicians and patients still prefer to perform the fresh embryo transfer, which can shorten the treatment time and time-to-live birth (TTLB). In this context, reducing the probability of PE occurrence on the hCG day during ovulation induction may be a more attractive option for IVF population. So some clinicians and scholars attempt to identify potential predictors related to PE occurrence. A recently published indicated that gonadotropin(Gn) dose, E2 on the hCG day, age, and many other factors were related to progesterone increase in multivariate regression models $[8,9]$. A retrospective study of a cohort of fresh IVF/ICSI cycles presented that Basal (Day 3 of the cycle) progesterone $(P)$ could significantly predict PE occurrence on the day of HCG in antagonist regimen [10]. However, the above studies did not to analyze whether the factor of different ovulation induction regimens was associated with the occurrence of PE.

We discovered that the progesterone level on the HCG administration was significantly difference in the same patients using different ovulation induction regiments during controlled ovulation stimulation (COS), so we speculated that the ovulation induction regiments may be related to the occurrence of PE. More resent a number of investigators used $0.9 \mathrm{ng} / \mathrm{ml}$ as a proper cut-off not previously thought to be $1.5 \mathrm{ng} / \mathrm{ml}$, because the researchers observed a detrimental effect on pregnancy rates as soon as progesterone levels exceed $0.9 \mathrm{ng} / \mathrm{ml}$ [11]. Given the above, the goal of this study was to develop a nomogram to explore the baseline indexes (including the ovulation induction regiments) to predict the occurrence of PE $(0.9 \mathrm{ng} / \mathrm{ml})$ on the day of HCG before IVF/ICSI treatment.

\section{Material And Methods}

\section{Patients}


The study project and the protocols were approved by the Ethics Committee of the Changzhou maternal and child health care hospital. The sighed informed consent was obtained from all patients. All the data in the study were collected from patients undergoing standard and routine IVF treatment.

Data (2698 cycles) in the retrospective analysis were collected from IVF cycles with a GnRH antagonist or agonist pituitary suppression protocol (GnRHa long Protocol and Ultra-long Protocol), that were performed in the IVF Unit of Changzhou maternal and child health care hospital in 2017-2019. Exlcusion criteria:(1)patient with chromosomal abnormal on either side of the couple;(2)Patients with ovarian cysts at the start of the ovarian stimulation procedure;(3)Patients with abnormal uterine cavity, ovarian deficiency and other organic disease; The fresh embryo transfer (ET) was cancelled in the case of increasing progesterone levels $(p \geq 1.0 \mathrm{ng} / \mathrm{ml})$.

\section{Ovarian stimulation}

Three protocols for controlled ovarian stimulation were performed based on age, anti-üllerian hormone (AMH) levels, number of antral follicles (AFC) and previous ovarian response, either recombinant FSH (Gonal F, Merck Serono, Geneva, Switzerland) or human menopausal gonadotrophin (HMG; 75 IU; Livzon Pharmaceutical, China) was used with daily doses between 150 and $300 \mathrm{IU}$. In the agonist long group, $0.1 \mathrm{mg}$ triptorelin (Decapeptyl, Ipsen, Paris, France) was administered for 7 days on menstrual cycle day 3 for inhibition of pituitary. In the ultra-long group, a long-acting gonadotrophin-releasing agonist (leuprorelin acetate, $3.75 \mathrm{mg}$, Lizhu Pharmaceutical Trading Co.) was administered on the menstrual cycle day 3 for pituitary inhibition. In the antagonist group, FSH/HMG was started on cycle day 3 and 0.25 cetrorelix (Cetrotide ${ }^{\circledR}$, Merck Serono, Geneva, Switzerland) was injected subcutaneously as a daily dose from the 6th day of stimulation until the day of oocyte maturation triggering. Adjustment of FSH/HMG dose was performed according to the ovarian response as assessed by ultrasonography and serum estradiol levels. When $\geq 3$ follicles in a mean diameters of $\geq 18 \mathrm{~mm}$ were present, HCG(5000IU urinary HCG and 250ug recombinant HCG) were administered to trigger final oocyte maturation. Oocyte retrieval was performed approximately $35 \mathrm{~h}-37 \mathrm{~h}$ later.

\section{In vitro fertilization and embryo culture}

Depending on the semen parameters, fertilization of the retrieved oocyte was performed in vitro by either conventional IVF or ICSI. Cleavage embryo was assessed for number and regularity of blastomeres and the degree of embryonic fragmentation; Grade I and Grade II embryos were top-quality embryos, the criteria were described previous.[12] Blastocyst embryo was evaluated for expansion degree and the number of inner cell mass and trophoblast cells on the basis of Garden scoring method. [13]

If the patient conditions were allowed, one good-quality blastocyst embryo on day 5(6) was selected for transfer.

\section{Hormonal analysis}


Serums sample were collected on the moring of menstrual cycle day 3 and the day of HCG trigger during ovarian stimulation. Serum FSH, LH, E2, and progesterone levels were measured routinely by commercially available kits (Abbott Biologicals B.V. The Netherlands) based on chemiluminescence. The lower limits of sensitivity were as follows: $\mathrm{FSH}=0.06 \mathrm{mlU} / \mathrm{ml}, \mathrm{LH}=0.09 \mathrm{mlU} / \mathrm{ml}, \mathrm{E} 2=10 \mathrm{pg} / \mathrm{ml}$ and $\mathrm{P}=$ $0.1 \mathrm{ng} / \mathrm{ml}$. Intra- and interassay precision rates at the concentrations, expressed as coefficients of variance were $3.8 \%$ and $6.4 \%$ for $\mathrm{LH}, 21.0 \%$ and $12.0 \%$ for E2 and $9.57 \%$ and $11.19 \%$ for progesterone respectively.

\section{PE definition}

Previously, it had been demonstrated that serum progesterone levels elevation on the day of hCG administration were associated with reduced ongoing pregnancy rates. Although most of the studies presented that progesterone's cut-off point for $P E$ is $1.5 \mathrm{ng} / \mathrm{ml}$, a meta-analysis including several thousand fresh IVF-ET cycles, observed a detrimental effect on pregnancy rates when the progesterone levels exceed $0.9 \mathrm{ng} / \mathrm{ml}$ on the HCG trigger day[11]. Present study also confirmed that the clinical pregnancy rate significantly decreased as soon as progesterone levels exceed $0.9 \mathrm{ng} / \mathrm{ml}(60.26 \otimes 50.56, P<0.05)$.For the above reason, $P E$ occurrence was defined as progesterone $\geq 0.9 \mathrm{ng} / \mathrm{mL}$. The population was divided into two groups: No PE group and PE group.

\section{Statistical analysis}

Two independent sample $t$-test, Nonparametric tests, and the Chi-squared test were performed to explore the distribution of baseline characteristics (ages, BMI, years of infertility et al.) and basal (day 3) hormonal profile between the two groups, and to predict the occurrence of PE after ovarian stimulation.

Logistic regression was performed to explore the multivariate model and to predict PE occurrence, and the regression models, the odds ratio (OR) and 95\% Confidence Intervals (CI) were estimated. Multivariate logistic regression analysis was used to generate coefficients for each variable and the constant in the equation. The predictive accuracy of the models was measured using the average optimism of the area under the curve (AUC), quantifying the level of agreement between the predicted probabilities and the actual possibility of having the event of interest. A Nomogram was constructed to be a graphic representation of the prediction model with the R software. Hosmer and Leme test checked for calibration and differentiation.

Data analysis was done with Statistical Package for Social Sciences (SPSS) version 26.0 (SPSS Incl., USA). The significance level for all statistical tests was $P \leq 0.05$.

\section{Results}

\section{General characteristics and basal hormonal profile}

2332 patients underwent 2689 IVF/ICSI cycles, and the PE occurrence rate was $30 \%$. There were 1881 cycles in the NPE group and 808 cycles in the PE group, respectively. There were no statistical differences 
in Female age, Duration of infertility, assisted reproductive treatment, Primary infertility, Baseline FSH, Baseline LH, Baseline E2 and Trigger day LH.(p all >0.05) (seen in Fig. 1).

BMI in the NPE group was significantly higher than that in the PE group. The distribution of ovulation induction protocols was significantly different between the two groups. Namely, PE occurrence rate is significantly different among the ovulation induction protocols, and the Antagonist protocol had a higher PE occurrence rate (51.4\%). Baseline P was higher in the PE group than that in the NPE group (8.31 \pm 7.97vs. $5.90 \pm 7.79 \mathrm{ug} / \mathrm{mL}, \mathrm{p}<0.0001$ ). There all were significantly different in $\mathrm{AMH}, \mathrm{Gn}$ dose, Gn days, Trigger day FSH, Trigger day LH and Trigger day E2 levels between the two groups (seen in Fig. 1).

There were no significant differences in the $2 \mathrm{PN}$ oocyte rate, Top-quality cleavage rate and blastocyst formation rate between the two groups. These indicated the increase of progesterone does not significantly detrimental effect the oocyte quality and embryo development potential, but the thickness of endometrial in PE group was obviously thinner and then it lead to a significant decline in clinical pregnancy rate and implanting rate. These suggested that the increase of progesterone negatively affects clinical outcome by affecting the endometrial not the oocyte quality.

\section{Variable and multivariable analyses the basic factors association with PE occurrence}

That released that number of cycles, BMI, Baseline P, AMH, AFC and ovulating induction protocols could significantly predict PE in total IVF/ICSI population. The multivariable regression analysis was performed through logistical regression, which included all significant variables (considering the collinearity of AFC and $\mathrm{AMH}, \mathrm{AFC}$ is excluded). In the total model, ovulating induction protocols, Baseline $\mathrm{P}$ and $\mathrm{BMI}$ were included as predictors. The model correlation regression coefficients and relevant parameters are presented in Fig. 2.

\section{Established the prediction models of $\mathrm{PE} \geq 0.9 \mathrm{ng} / \mathrm{ml}$ and tested its differentiation and calibration}

On the basis of the results of univariate and multivariate logistic regression analyses, a nomogram incorporating the significant factors was established to predict the PE occurrence on the HCG administration.(Fig. 3A)

The contribution degree of independent predictors (ovulating induction protocols, Baseline $\mathrm{P}$ and $\mathrm{BMI}$ ) to the prediction model matched the corresponding score, and the sum of the matching score of each factor was converted into the probability of PE occurrence, on this basis the clinical prediction model of PE occurrence was established. The calibration curves for PE occurrence showed good calibration. The model showed an AUC of $0.639((95 \% \mathrm{Cl} 0.596 \sim 0.681), \mathrm{P}=0.000)$. Hosmer and Lemeshow test were performed for evaluating both calibration and discrimination and confirmed our nomogram is a userfriendly graphical representation of the model $\left(\chi^{2}=4.750 \otimes P=0.784\right)$. ( Fig. 3B.C)

\section{Discussion}


The predictive model confirmed that the combination of ovulation induction regimen with $\mathrm{BMI}$ and Basal $P$ could predict the PE occurrence on the HCG trigger day. Performance was evaluated using both calibration and discrimination, our nomogram is a user-friendly graphical representation of the model. We hypothesize that the nomogram can be used in routine practice to facilitate physicians to avoid the occurrence of PE by evaluating the basic characteristics of patients. For example the doctors performed the ovulation induction regimen for patient before IVF/ICSI treatment, if the BMI of the patient is lower and Basal $P$ value is higher, doctors should avoid selecting antagonist program. If the patients had to be performed the antagonist program for some factors, the physician should carefully control the dosage of $\mathrm{Gn}$ and the appropriate amount of follicular when the score is high as assessed by the nomogram.

The occurrence of PE on the day HCG has been reported between $6 \%$ and $30 \%$ in controlled ovarian induction cycles [14]. The high incidence of $\mathrm{PE}$ is about $30 \%$ in present study, which may be due to the low cut-off value of PE $(0.9 \mathrm{ng} / \mathrm{ml})$. To our knowledge, the present study is the first retrospective cohort trial to evaluate whether the ovulation induction regimens is associated with the PE occurrence. The rates of $P E$ varied greatly in different ovarian induction schemes and the rate of $P E$ in the antagonist regimen was significantly higher than that the agonist regimen in our study. (51.4\%\&24.4\%).Many previous studies $[15,16]$ have also reported that the progesterone level of the antagonist protocol was higher than that of $\mathrm{GnRHa}$ long protocol. The GnRH agonist generally was used to the patients with higher or lower ovarian response (such as PCOS) and the GnRH antagonist was used primarily to the patients with regular ovarian response in most of centers. It was speculated that there were more dominant follicles and higher E2 on the HCG trigger days in high responders than the normal responders, this hypothesis was in keeping with the observation [17]. Another possibility is that the effect of endogenous LH was prevented by the extended down-regulation of pituitary $\mathrm{GnRH}$ receptors induced by $\mathrm{GnRH}$ agonists during COS, but there was no down-regulation of pituitary during COS, the higher endogenous $\mathrm{LH}$ in the GnRH antagonist regimen promoted the production of progesterone level [18].

In this study, Basal P is association with the occurrence of PE on the HCG day,the capacity of basal P to predict the PE occurrence has also been confirmed in previous study, only including GnRH antagonist[10]. The present study including three kinds of $\mathrm{GnRH}$ confirmed these findings in much larger dataset $(\mathrm{n}=$ 2689 cycles), hence providing increased statistical power. However, it is not clear that why basal $P$ is closely associated with PE on the day of HCG. It might be assumed that basal P mainly reflects progesterone by the adrenal, Fanchin R et.al[19] discovered that adrenal suppression lowered progesterone levels from values achieved after $\mathrm{GnRH}$ analogue. Yet, the net increases in progesterone were unaltered during COS, thereby confirmed that the adrenal contribution the circulating levels of baseline hormone. In fact, the PE results solely from an effect of exogenous gonadotropins on the ovary during the COS[23]. In our results, it was also confirmed that the amount of gonadotropins administered and the number of mature follicles by COS were associated with the PE occurrence (supplement 1 ).

Another factor influencing the PE occurrence is $\mathrm{BMI}$, our data showed that PE is more likely to occur in patients with lower $\mathrm{BMI}$ than the patients with higher $\mathrm{BMI}$, the reason may be that $\mathrm{BMI}$ is associated with ovarian response. As previous reports discovered that obese patients required more $\mathrm{Gn}$ than thin women 
and the response of thin patients was in excess of the thin women in terms of E2 levels and number of oocytes retrieved, higher serum E2 levels and number of oocytes retrieved were associated with elevated progesterone concentrations[20]. However, the explanation for the need more total $\mathrm{Gn}$ in obese women is not clear at present. Presumably women with higher BMI also have more fat cells in their bodies whose content is positively correlated with leptin and Leptin has a stimulating effect on GnRH pulse. High levels of leptin may have an anti-Gn effect and reduce the absorption of $\mathrm{Gn}$, and then increase the $\mathrm{Gn}$ amount required for oocyte maturation and development [24].

High progesterone levels above a certain threshold could reduce the clinical pregnancy rate [1]. However, whether $\mathrm{PE}$ is bad for the embryo or the endometrium is controversial, our results showed that $\mathrm{PE}$ on the HCG day did not negatively affect the embryo quality, and PE negatively affected the thickness of the endometrium. Some authors have proposed to confirm the consequences of high progesterone levels during the end follicular phase on the histologic transformations of the endometrium [22]. Direct evidence for an effect of PE on the endometrium has been provided by endometrial gene expression analysis $[4,5]$. So, PE may cause asynchrony between the developing embryo and endometrium. Some reports also supported that the elevation of plasma progesterone levels is to advance secretory transformations of endometrium and modify the timing of endometrium receptivity (window of implantation) [21].

As for the limitations and disadvantages, this study is a retrospective and single-center study with certain limitations. Although the remarkable and the most critical confounders were well controlled through the multivariable regression analysis, the unknown residual still existed, and the bias cannot be excluded completely. The collected data was from a single center and a period of only three years. The model was established based on vary limited predictors obtained before an IVF/ICSI treatment. PE occurrence is a dynamic and complex endocrine process and we appreciate successful predicting PE depends on more than the factors in this model alone. Therefore, prospective, large-scale and multicenter clinical trials should be carried out in future.

Despite these limitations, our results suggest that our nomogram predicting the PE occurrence on the HCG trigger day could be a useful tool in helping physicians and infertile women to avoid PE before IVF/ICSI treatment. We highlight that the PE occurrence on the HCG administration could be relatively high basal $\mathrm{P}$, low BMI and using antagonist regimen during COS.

\section{Conclusion}

In conclusion, an objective and accurate prediction nomogram model for the PE occurrence on the HCG administration was drawn up in our study. The new nomogram model has proved to be a novel tool and easy to use to predict the PE occurrence on the HCG administration. Furthermore, it was able to select appropriate ovulation regiment for infertility patients by evaluating the basal $\mathrm{P}$ and $\mathrm{BMI}$ and it can be avoided the PE occurrence by adjusting the total $\mathrm{Gn}$ dosage and the number of the dominant follicles. But not least, large-scale and multicenter clinical trials will provide more robust evidence to our results. 


\section{Abbreviations}

PE: progesterone elevation; HCG: human chorionic gonadotrophin; COS: Controlled ovulation stimulation; AUC: Area under the curve; TTLB: Time-to-live birth; Gn: gonadotropin; ET: Embryo transfer; AMH: Antiüllerian hormone; AFC: Antral follicles; HMG: Human menopausal gonadotrophin; OR: odds ratio; $\mathrm{Cl}$ : Confidence Intervals; SPSS: Statistical Package for Social Sciences; BMI: Body Mass Index; FSH: Follicle stimulating hormone; GnRH: Gonadotropin releasing hormone; LH: Luteinizing hormone.

\section{Declarations}

\section{Ethics approval and consent to participate:}

The study project and the protocols were approved by the Ethics Committee of the Changzhou maternal and child health care hospital (17020490718). The sighed informed consent was obtained from all patients. All the data in the study were collected from patients undergoing standard and routine IVF treatment.

\section{Consent for publication:}

Not applicable.

\section{Availability of data and material:}

The primary data for this study is available from the authors upon direct request.

\section{Competing interests:}

The authors declare that they have no competing interests.

\section{Funding:}

This project is funded by the basic \& applied research project of Changzhou (CJ20179049) and special fund for clinical research of Chinese Medical Association.

\section{Authors' contributions:}

Chun-Mei Yu, Chao-Zhou, Xiu-Liang Dai, Jian-Mei Zhou, Hai-Yan Yang, Yu-Feng Wang and Li Chen contributed in conception, design, statistical analysis and drafting the manuscript. All authors approved the final version for submission.

\section{Acknowledgements:}

This study was supported by the Affiliated Changzhou Maternal and Child Health Care Hospital of Nanjing Medical University. We thank the members of the IVF Unit of the Affiliated Changzhou Maternal and Child Health Care Hospital. 


\section{Conflicts of interest:}

The authors declare that they have no conflicts of interest related to the subject matter or materials discussed in this article.

\section{References}

1. Venetis CA, Kolibianakis EM, Bosdou JK, Tarlatzis BC. Progesterone elevation and probability of pregnancy after IVF: a systematic review and meta-analysis of over 60000 cycles. Hum Reprod Update 2013;19: 433 - 457

2. Martínez F., Coroleu B., Clua E., et al. Serum progesterone concentrations on the day of HCG administration cannot predict pregnancy in assisted reproduction cycles [J]. Reproductive biomedicine online, 2004, 8(2): 183-190.

3. Venetis C. A., Kolibianakis E. M., Papanikolaou E., et al. Is progesterone elevation on the day of human chorionic gonadotrophin administration associated with the probability of pregnancy in in vitro fertilization? A systematic review and meta-analysis [J]. Human reproduction update, 2007, 13(4): 343-355.

4. Campos C.C., Hartling I., Kaur M., et al. Intramammary infusion of lipopolysaccharide promotes inflammation and alters endometrial gene expression in lactating Holstein cows [J]. Journal of dairy science, 2018, 101(11): 10440-10455.

5. Lawrenz B., Fatemi H. M. Effect of progesterone elevation in follicular phase of IVF-cycles on the endometrial receptivity [J]. Reproductive biomedicine online, 2017, 34(4): 422-428.

6. Evans J., Hannan N. J., Edgell T. A., et al. Fresh versus frozen embryo transfer: backing clinical decisions with scientific and clinical evidence [J]. Human reproduction update, 2014, 20(6): 808-821.

7. Roque M. Freeze-all policy: is it time for that? [J]. Journal of assisted reproduction and genetics, 2015, 32(2): 171-176.

8. Venetis C. A., Kolibianakis E. M., Bosdou J. K., et al. Progesterone elevation and probability of pregnancy after IVF: a systematic review and meta-analysis of over 60000 cycles [J]. Human reproduction update, 2013, 19(5): 433-457.

9. Saleh H. A., Omran M. S., Draz M. Does subtle progesterone rise on the day of HCG affect pregnancy rate in long agonist ICSI cycles? [J]. Journal of assisted reproduction and genetics, 2009, 26(5): 239242.

10. Venetis CA, Kolibianakis EM, Bosdou JK, Lainas GT, Sfontouris IA, Tarlatzis BC, Lainas TG. Basal serum progesterone and history of elevated progesterone on the day of hCG administration are significant predictors of late follicular progesterone elevation in GnRH antagonist IVF cycles. Hum Reprod. 2016 Aug;31(8):1859-65.

11. Fanchin R, de Ziegler D, Taieb J, Hazout A, Frydman R. Premature elevation of plasma progesterone alters pregnancy rates of in vitro fertilization and embryo transfer. Fertil Steril 1993;59:1090-4. 
12. Tournaye H, Sukhikh GT, Kahler E, Griesinger G. A Phase III randomized controlled trial comparing the efficacy, safety and tolerability of oral dydrogesterone versus micronized vaginal progesterone for luteal support in in vitro fertilization. Hum Reprod. 2017 May 1;32(5):1019-1027. doi:

10.1093/humrep/dex023. Erratum in: Hum Reprod. 2017 Oct 1;32(10):2152.

13. Gardner DK, Schoolcraft WB. In virto culture of human blastocyst. In Janson R, Mortimer D (eds). Towards Reproductive Certainty: Infertility and Genetics Beyond 1999. Carnforth: Parthenon Press, 1999, 378-388.

14. Bosch E., Labarta E., Crespo J., et al. Circulating progesterone levels and ongoing pregnancy rates in controlled ovarian stimulation cycles for in vitro fertilization: analysis of over 4000 cycles [J]. Human reproduction (Oxford, England), 2010, 25(8): 2092-2100.

15. Lainas T. G., Petsas G. K., Zorzovilis I. Z., et al. Initiation of GnRH antagonist on Day 1 of stimulation as compared to the long agonist protocol in PCOS patients. A randomized controlled trial: effect on hormonal levels and follicular development [J]. Human reproduction (Oxford, England), 2007, 22(6): 1540-1546.

16. Orvieto R., Nahum R., Meltzer S., et al. GnRH agonist versus GnRH antagonist in ovarian stimulation: the role of elevated peak serum progesterone levels [J]. Gynecological endocrinology : the official journal of the International Society of Gynecological Endocrinology, 2013, 29(9): 843-845.

17. Bosch E, Labarta E, Crespo J, Simon C, Remohí J, Jenkins J, et al. Circulating progesterone levels and ongoing pregnancy rates in controlled ovarian stimulation cycles for in vitro fertilization: analysis of over 4000 cycles. Hum Reprod 2010;25:2092-100.

18. Schoolcraft W, Sinton E, Schlenker T, Huynh D, Hamilton F, Meldrum DR.Lower pregnancy rate with premature luteinization during pituitary suppression with leuprolide acetate. Fertil Steril 1991;55:563-6.

19. Fanchin R, Righini C, Olivennes F, Taieb J, de Ziegler D, Frydman R. Premature plasma progesterone and androgen elevations are not prevented by adrenal suppression in in vitro fertilization. Fertil Steril 1997;67:115-9

20. Maged AM, Fahmy RM, Rashwan H, Mahmood M, Hassan SM, Nabil H, Hany A, Lotfy R, Lasheen YS, Dahab S, Darwish M. Effect of body mass index on the outcome of IVF cycles among patients with poor ovarian response. Int J Gynaecol Obstet.2019Feb;144(2):161-166.

21. Fanchin R, de Ziegler D, Taieb J, Hazout A, Frydman R. Premature elevation of plasma progesterone alters pregnancy rates of in vitro fertilization andembryo transfer. Fertil Steril 1993;59:1090-4.

22. Chetkowski RJ, Kiltz RJ, Salyer WR. In premature luteinization, progesterone induces secretory transformation of the endometrium without impairment of embryo viability. Fertil Steril 1997;68:292-7.

23. Adda-Herzog E, Poulain M, de Ziegler D, Ayoubi JM, Fanchin R. Premature progesterone elevation in controlled ovarian stimulation: to make a long story short. Fertil Steril. 2018 Apr;109(4):563-570.

24. Pasquali R, Pelusi C, Genghini S, Cacciari M, Gambineri A. Obesity and reproductive disorders in women. Hum Reprod Update. 2003 Jul-Aug;9(4):359-72. 


\section{Figures}

\begin{tabular}{|c|c|c|c|}
\hline Variables & NPE group $(n=1881)$ & $\mathrm{PE}$ group $(\mathrm{n}=808)$ & $P$ \\
\hline Age (years) & $30.36 \pm 3.76$ & $30.40=3.96$ & 0.83 \\
\hline $\mathrm{BMI}(\mathrm{kg} / \mathrm{m} 2)$ & $23.21 \pm 7.74$ & $22.15 \pm 3.48$ & $<0.0001^{*}$ \\
\hline Duration of infertility (years) & $3.37 \pm 2.30$ & $3.33 \pm 2.35$ & 0.48 \\
\hline \multicolumn{4}{|l|}{ OIP, n (\%) } \\
\hline $\mathrm{GnRHa}$ long protocol & $1319(70.12)$ & $424(52.47)$ & $<0.0001^{*}$ \\
\hline Ultra-long protocol & $294(15.63)$ & $100(12.37)$ & 0.032 \\
\hline Antagonist protocol & $269(14.30)$ & $284(35.14)$ & $<0.0001^{*}$ \\
\hline \multicolumn{4}{|l|}{$\mathrm{ART}, \mathrm{n}(\%)$} \\
\hline ICSI & $216(11.48)$ & $96(11.88)$ & \\
\hline IVF & $1546(82.19)$ & $657(81.31)$ & 0.58 \\
\hline Primary infertility, $n(\%)$ & $1055(56.08)$ & $455(56.31)$ & 0.93 \\
\hline Baseline FSH (IU/L) & $6.33(5.41,7.43)$ & $6.23(5.40,7.38)$ & 0.46 \\
\hline Baseline LH(TU/L) & $5.76 \pm 4.38$ & $5.51 \pm 4.31$ & 0.13 \\
\hline Baseline E2 (ng/L) & $31.01(22.47,41.48)$ & $32.38(23.01,42.85)$ & 0.047 \\
\hline Baseline P (ng/ml) & $5.90 \pm 7.79$ & $8.31 \pm 7.97$ & $<0.0001^{*}$ \\
\hline $\mathrm{AMH}(\mu \mathrm{g} / \mathrm{dL})$ & $3.97(2.47,6.04)$ & $3.38(2.08,5.38)$ & $<0.0001^{*}$ \\
\hline Gn dose (IU) & $1500(1125,2075)$ & $1875(1325,2588)$ & $<0.0001^{*}$ \\
\hline Gn days (days) & $9.05 \pm 1.96$ & $9.45 \pm 2.02$ & $<0.0001^{*}$ \\
\hline Trigger day $\mathrm{FSH}(\mathrm{IU} / \mathrm{L})$ & $10.35 \pm 5.13$ & $12.84 \pm 5.53$ & $<0.0001^{*}$ \\
\hline Trigger day E2 (ng/L) & $3210 \pm 1785$ & $3869 \pm 1848$ & $<0.0001^{*}$ \\
\hline Trigger day $\mathrm{LH}(\mathrm{IU} / \mathrm{L})$ & $2.06(1.37,3.02)$ & $1.96(1.27,2.92)$ & 0.088 \\
\hline Trigger day $\mathrm{P}(\mathrm{ng} / \mathrm{mL})$ & $0.59(0.46,0.73)$ & $1.13(1.00,1.36)$ & $<0.0001^{*}$ \\
\hline The thickness of endometrial $(\mathrm{mm})$ & $12.02 \pm 2.42$ & $11.58 \pm 2.49$ & $<0.001^{*}$ \\
\hline NO of oocytes retrieved $(n)$ & $10.0(7.0,14.0)$ & $11.0(8.0,14.0)$ & $<0.0001^{*}$ \\
\hline $2 \mathrm{PN}$ oocytes rate $(\%)$ & $14065 / 18267(76.99)$ & $6546 / 8516(76.86)$ & 0.82 \\
\hline Top-quality cleavage rate (\%) & $10261 / 15965(64.08)$ & $4685 / 7385(63.44)$ & 0.22 \\
\hline Blastocyst formation rate $(\%)$ & $6369 / 9007(70.71)$ & $2777 / 3979(69.79)$ & 0.29 \\
\hline Fresh embryo transfer rate, $n(\%)$ & $818 / 1881(43.49)$ & $180 / 808(22.0)$ & $<0.0001^{*}$ \\
\hline Clinical ongoing pregnancy rate (\%) & $493 / 818(60.26)$ & $91 / 180(50.56)$ & $0.019^{*}$ \\
\hline Implanting rate (\%) & $621 / 1398(44.42)$ & $117 / 306(38.23)$ & $0.048^{*}$ \\
\hline
\end{tabular}

Figure 1

Comparison of variables between cycles with and without PE囚 $\geq 0.9 \mathrm{ng} / \mathrm{ml} 囚$ on the day of HCG administration. OIP: Ovulation induction protocol; ART: Assisted reproductive treatment 
Univariable and Multivariable regression analyses of the association between baseline variables and the occurrence of PE ( $\geqslant 0.9 \mathrm{ng} / \mathrm{ml}$ ) on the day of hCG.

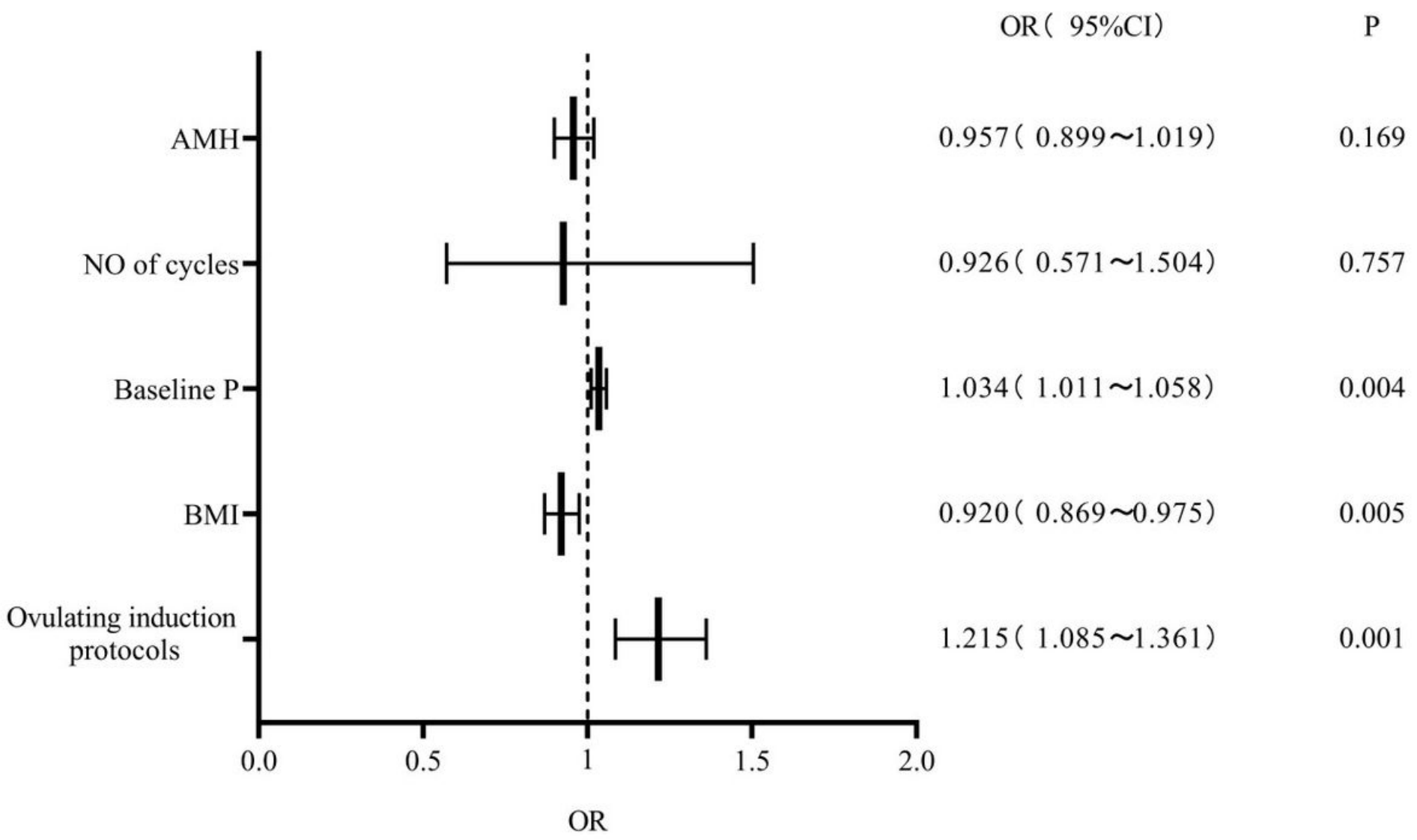

Figure 2

Univariable and Multivariable regression analyses of the association between baseline variables and the occurrence of PE ( $\geq 0.9 \mathrm{ng} / \mathrm{ml})$ on the day of hCG administration. Cl: Correlation confidence
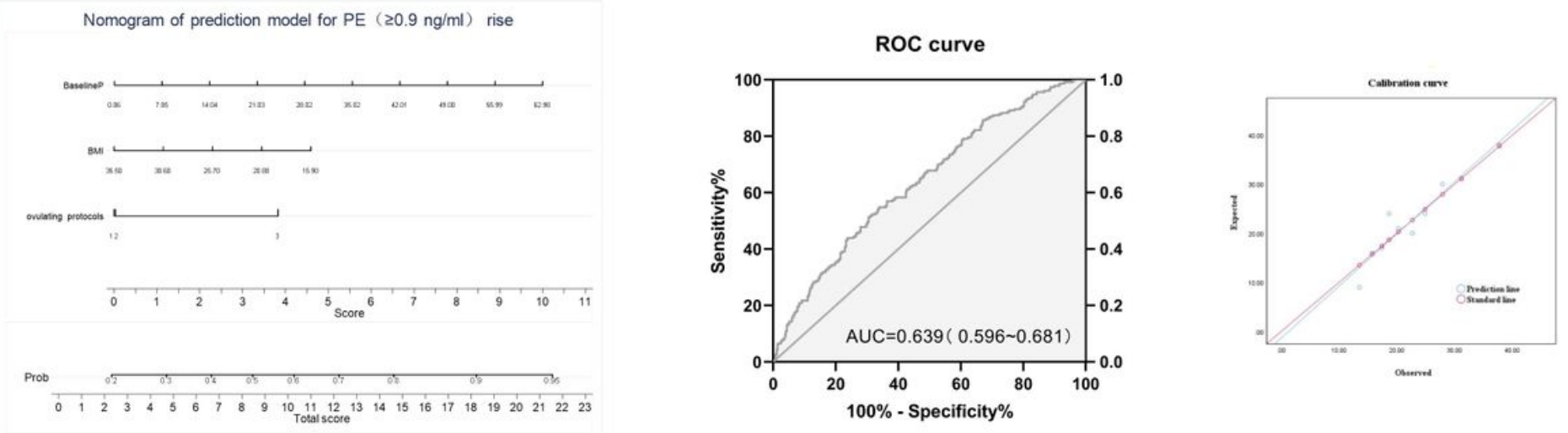

a

b

c

Figure 3 
Nomogram to predict the probability of the occurrence of $P E \unrhd \geq 0.9 \mathrm{ng} / \mathrm{ml} \rrbracket$ on the $H C G$ administration. A. The probability of PE is calculated by drawing a line to the point on the axis for each of the following variables: basal P, BMI and the type of ovulating protocol. The points for each variable are summed and located on the total point line. Next, a vertical line is projected from the total point line to the predicted probability bottom scale to obtain the individual probability of PE.囚ovulating protocols: 1, GnRHa long protocol; 2, Ultra-long protocol; 3 , Antagonist protocol $\mathbb{B}$ B. Discrimination for the training cohort. ROC curve of the model with an AUC of 0.639 (95\% Cl: 0.596- 0.681). C. Calibration for the training cohort

\section{Supplementary Files}

This is a list of supplementary files associated with this preprint. Click to download.

- SupplmentFigure1.jpg 